\title{
Bis-imidazolinylindoles are active against methicillin-resistant Staphylococcus aureus and multidrug-resistant Mycobacterium tuberculosis
}

\author{
Rekha G Panchal ${ }^{1}$, Douglas Lane ${ }^{2}$, Helena I Boshoff ${ }^{3}$, Michelle M Butler ${ }^{4}$, Donald T Moir ${ }^{4}$, \\ Terry L Bowlin ${ }^{4}$ and Sina Bavari ${ }^{1}$
}

The Journal of Antibiotics (2013) 66, 47-49; doi:10.1038/ja.2012.93; published online 14 November 2012

Keywords: antibacterials; antibiotic resistance; bis-imidazolinylindoles; broad-spectrum

The increasing prevalence of methicillin-resistant Staphylococcus aureus (MRSA) ${ }^{1}$ and multi/extensively drug-resistant Mycobacterium tubcerulosis is a major global health problem., ${ }^{2,3}$ The development of a new class of antibacterials based on a novel chemotype or mechanism of action, and with efficacy against both susceptible and resistant strains, would be an extremely useful addition to current therapeutic options.

Previously, we identified four novel bis-imidazolinylindole compounds MBX 1066, MBX 1090, MBX 1113 and MBX 1128, that represent a new antibacterial chemotype with potent activity against a broad spectrum of both Gram-positive and Gram-negative bacterial species. ${ }^{4}$ While these compounds are rapidly bactericidal, their precise bacterial target is unknown, but studies suggest that they bind to DNA and inhibit DNA synthesis. In vitro, these compounds exhibit potent activity against antibiotic-resistant strains such as ciprofloxacin-resistant $B$. anthracis Ames, MRSA and vancomycinresistant enterococci. Members of this class of compounds also exhibit activity in vivo in mouse models of lethal infection with B. anthracis, Y. pestis and methicillin-susceptible S. aureus. ${ }^{4}$

A particularly important feature of this new chemotype is the very low potential for development of mutations to resistance. Specifically, no mutations to resistance were observed for MBX 1066 and MBX 1113 and MBX 1162, even after 20 days of growth of S. aureus ${ }^{4,5}$ and Escherichia coli (unpublished data) in sublethal concentrations. However, for MBX 1090, mutant clones were able to grow in concentrations up to $16 \times$ the compound MIC. ${ }^{4}$ Interestingly, the mutants were not cross-resistant to the other members of this class of compounds and the mutations responsible for MBX 1090 resistance were demonstrated to cause elevated production of an efflux pump for which other compounds in this class are not a substrate. ${ }^{5}$ In this study, we extended the analysis of the activities of the bis-imidazolinylindole class of antibacterials against two clinically important antibiotic-resistant pathogens.

As our previous studies demonstrated that the bis-imidazolinylindole compounds MBX 1066, MBX 1090, MBX 1113 and MBX 1128 (see Table 1 for the structures) are active against M. smegmatis, ${ }^{4}$ we investigated their effect on one susceptible (H37Rv) and seven multidrug-resistant isolates of $M$. tuberculosis. Table 1 summarizes the in vitro activities of the four compounds. MICs ranged from $<0.02$ to $1.3 \mu \mathrm{g} \mathrm{ml}^{-1}$. The compounds MBX 1066 and MBX 1113 exhibited more potent antimycobacterial activity than did MBX 1090 and MBX 1128. Next, the antimycobacterial activity of MBX 1066 was compared with its structural analog, MBX 1162 (see Table 2 for the structure), which has previously shown to exhibit potent activity against Gram-positive and Gram-negative bacteria. ${ }^{6}$ As shown in Table 2, MBX 1162 was highly active against all the multidrugresistant isolates of $M$. tuberculosis and compared favorably with MBX 1066. MBX 1162 was assayed for cytotoxicity against HeLa cells as described previously ${ }^{4}$ and exhibited slightly increased toxicity $\left(\mathrm{CC}_{50}=4 \mu \mathrm{g} \mathrm{ml}^{-1}\right)$ compared with its parent MBX 1066 $\left(\mathrm{CC}_{50}=33 \mu \mathrm{g} \mathrm{ml}^{-1}\right){ }^{4}$

Previously, we reported that the four bis-imidazolinylindole compounds examined herein are quite active in vitro against MRSA, with MIC values ranging from 0.2 to $5 \mu \mathrm{g} \mathrm{ml}{ }^{-1}$. To investigate the in vivo efficacy of these compounds, ICR/Swiss Webster mice $(n=10)$ were challenged via the i.p. route with $S$. aureus strain NRS384, a community-associated methicillin-resistant (cMRSA) clinical isolate. One hour post infection, mice were treated via the i.v. route with a single dose of compound MBX 1066 (1 or $10 \mathrm{mg} \mathrm{kg}^{-1}$ ), MBX 1090 ( 1 or $10 \mathrm{mg} \mathrm{kg}^{-1}$ ) or MBX 1162

${ }^{1}$ Target Discovery and Experimental Microbiology Department, United States Army Medical Research Institute of Infectious Diseases, Frederick, MD, USA; ${ }^{2}$ Target Structure Based Drug Discovery Group, SAIC-Frederick, Inc., NCI-Frederick, Frederick, MD, USA; ${ }^{3}$ Tuberculosis Research Section, LCID, NIAID, NIH, 9000 Rockville Pike, Bethesda, MD, USA and ${ }^{4}$ Microbiotix Inc., One Innovation Dr., Worcester, MA, USA

Correspondence: Dr RG Panchal, Target Discovery and Experimental Microbiology Department, United States Army Medical Research Institute of Infectious Diseases, 1425 Porter Street, Fort Detrick, Frederick, MD 21702-5011, USA.

E-mail: rekha.panchal@amedd.army.mil

Received 19 April 2012; revised 22 August 2012; accepted 8 October 2012; published online 14 November 2012 
Table 1 Comparison of the MICs of bis-imidazolinylindole compounds against antibiotic-sensitive and multidrug-resistant isolates of M. tuberculosis

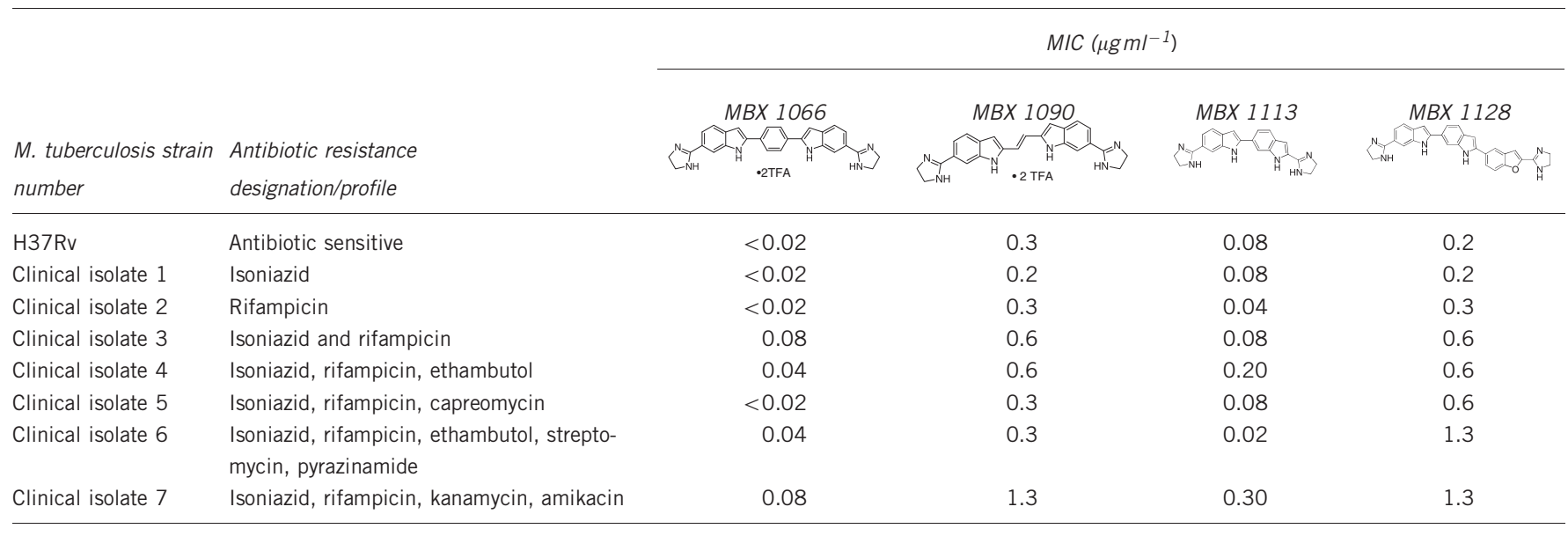

Table 2 MICs of the two most-potent bis-imidazolinylindole compounds against Beijing antibiotic-sensitive and multidrug-resistant clinical isolates of $M$. tuberculosis

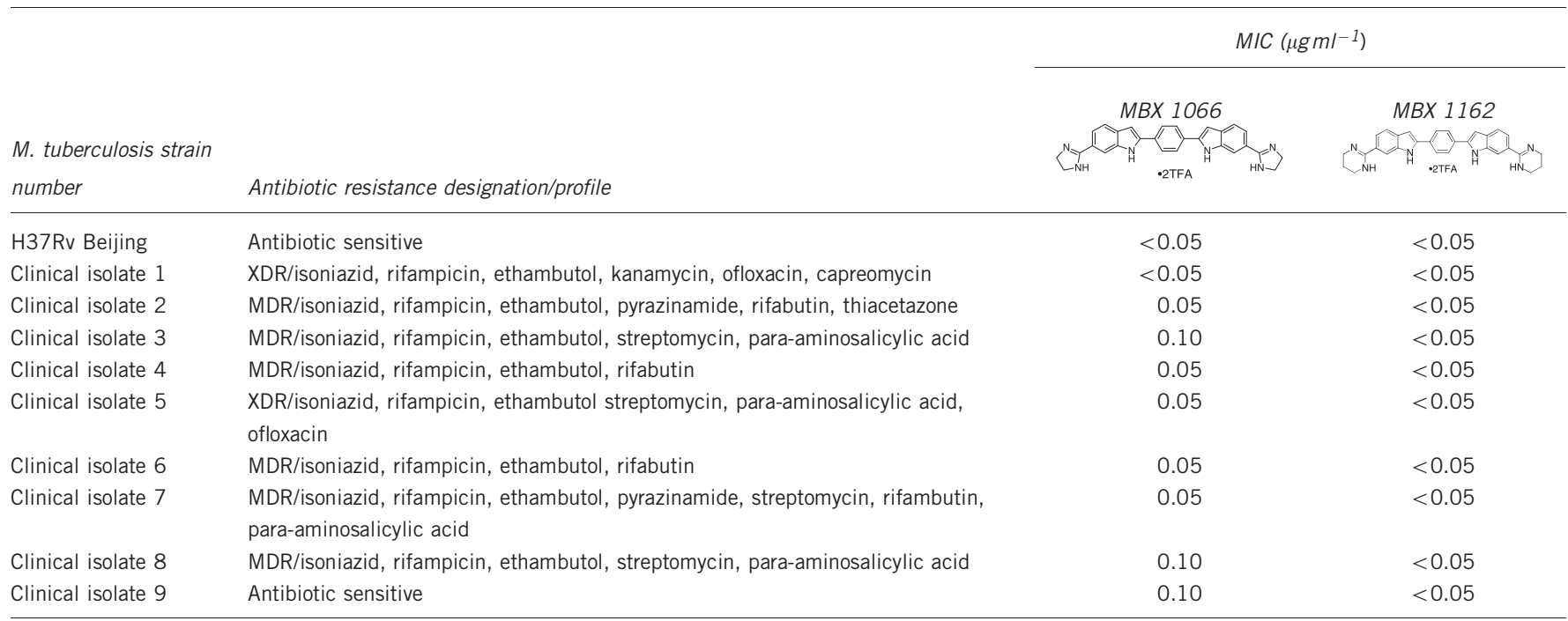

Abbreviations: MDR, multi drug-resistant; XDR, extensively drug-resistant.

Table 3 In vivo efficacy of bis-imidazolinylindole compounds against community-associated methicillin-resistant $S$. aureus

\begin{tabular}{|c|c|c|c|}
\hline Treatment & Dose $\left(m g k^{-1}\right)$ & $\begin{array}{c}\% \text { Survival } \\
(\mathrm{n}=10 \text { mice per group) }\end{array}$ & $\begin{array}{l}\text { Log reduction in CFU } \\
\text { from untreated control }\end{array}$ \\
\hline Vehicle control & 0 & 30 & - \\
\hline MBX 1066 & 1 & 90 & 3.9 \\
\hline MBX 1066 & 10 & 80 & 3.7 \\
\hline MBX 1090 & 1 & 90 & 3.6 \\
\hline MBX 1090 & 10 & 100 & 4.3 \\
\hline MBX 1162 & 1 & 90 & 3.5 \\
\hline MBX 1162 & 10 & 70 & 2.1 \\
\hline Daptomycin & 10 & 100 & 4.6 \\
\hline
\end{tabular}

(1 or $10 \mathrm{mg} \mathrm{kg}^{-1}$ ). A $70-100 \%$ survival rate was observed in the compound-treated mice (Table 3). The protective effects were similar to those observed with the reference antibiotic daptomycin. Furthermore, sampling of the spleen for bacterial load revealed a 2-4 log reduction in CFU (per tissue) in each of the groups compared with the untreated control (Table 3). Thus, the compounds are active in vivo against cMRSA infection.

In summary, we have shown that several members of the bisimidazolinylindole compounds exhibit very potent in vitro activity against both susceptible and multidrug-resistant isolates of M. tuberculosis as well as in vivo activity against cMRSA. A newer analog, MBX 1162, demonstrated equivalent potency and slightly increased mammalian cell toxicity compared with MBX 1066. Optimization of the scaffold proceeds with the goal of lessening toxicity while 
maintaining potency. These compounds represent a promising new scaffold for the treatment of drug-resistant bacterial pathogens.

\section{EXPERIMENTAL PROCEDURE}

\section{Bacterial strains}

The M. tuberculosis clinical isolates tested in this study are maintained at an inhouse repository at the Southern Research Institute (Table 1) and the National Institute of Allergy and Infectious diseases ${ }^{7}$ (Table 2). The drug resistance profiles for each clinical isolate are listed in their respective tables. S. aureus NRS384, a cMRSA clinical isolate representative of the USA300 clone, was used in the in vivo efficacy studies. The isolate is both staphylococcal chromosomal cassette mec type IV positive and Panton-Valentine leucocidin positive. The isolate was obtained from the Network on Antimicrobial Resistance in S. aureus (NARSA, Herndon, VA, USA).

\section{In vitro growth inhibition studies}

MIC was determined using the broth microdilution method. ${ }^{8}$ Briefly, compounds were dissolved in dimethyl sulfoxide and then diluted with Middlebrook 7H9 broth (BD, Sparks, MD, USA). Twofold serially diluted compounds $(0.1 \mathrm{ml}$ per well) were added to 96 -well microtiter plates. The plates were inoculated with appropriate bacterial cultures that were standardized turbidimetrically to a concentration of $5 \times 10^{5}$ per well. The plates were incubated at $37^{\circ} \mathrm{C}$ for 7 days. MIC was determined visually as the lowest amount of drug that completely inhibited growth and was scored by comparing the growth in each well with growth in the negative-control wells (bacteria only) and positive-control wells (isoniazid).

\section{In vivo efficacy in the mouse model of methicillin-resistant S. aureus infection}

Female ICR/Swiss mice (Charles River Laboratories, Wilmington, MA, USA) were challenged i.p. with $7.4 \times 10^{7} \mathrm{CFU}$ of cMRSA. After $1 \mathrm{~h}$, mice were treated via i.v. route with 1 or $10 \mathrm{mg} \mathrm{kg}^{-1}$ of compounds. On day 2 , mice that survived challenge were euthanized and spleen harvested. Homogenized spleen was serially diluted in sterile phosphatase-buffered saline and appropriate concentrations plated on Trypticase Soy Agar plates. The number of CFU per tissue was determined by quantitative colony counts.

\section{ACKNOWLEDGEMENTS}

This project has been funded in part by HDTRA1-06-C-0042 to Microbiotix Inc. and by Defense Threat Reduction Agency (DTRA) to RGP and SB, and with federal funds from the National Cancer Institute, National Institutes of Health (under contract N01-CO-12400) and in part by the Intramural Research Program of the NIH, NIAID. The content of this publication does not necessarily reflect the views or policies of the Department of Health and Human Services, nor does mention of trade names, commercial products or organizations imply endorsement by the US Government. This research was supported in part by the Developmental Therapeutics Program in the Division of Cancer Treatment and Diagnosis of the National Cancer Institute. Opinions, interpretations, conclusions and recommendations are those of the authors and are not necessarily endorsed by the US Army.

1 Grundmann, H., Aires-de-Sousa, M., Boyce, J. \& Tiemersma, E. Emergence and resurgence of meticillin-resistant Staphylococcus aureus as a public-health threat. Lancet 368, 874-885 (2006).

2 Anti-tuberculosis drug resistance in the world. Fourth global report. Vol. 394 The WHO IUATLD Global Project on Antituberculosis Drug Resistance Surveillance (World Health Organization, Geneva, WHO/HTM/TB 10-12, 2008).

3 Alexander, P. E. \& De, P. The emergence of extensively drug-resistant tuberculosis (TB): TB/HIV coinfection, multidrug-resistant TB and the resulting public health threat from extensively drug-resistant TB, globally and in Canada. Can. J. Infect. Dis. Med. Microbiol. 18, 289-291 (2007).

4 Panchal, R. G. et al. Novel broad-spectrum bis-(imidazolinylindole) derivatives with potent antibacterial activities against antibiotic-resistant strains. Antimicrob. Agents Chemother. 53, 4283-4291 (2009).

5 Opperman, T. J. et al. Efflux-mediated bis-indole resistance in Staphylococcus aureus reveals differential substrate specificities for MepA and MepR. Bioorg. Med. Chem. 18, 2123-2130 (2010).

6 Butler, M. M. et al. Comparative in vitro activity profiles of novel bis-indole antibacterials against gram-positive and gram-negative clinical isolates. Antimicrob. Agents Chemother. 54, 3974-3977 (2010).

7 Jeon, C. Y. et al. Extensively drug-resistant tuberculosis in South Korea: risk factors and treatment outcomes among patients at a tertiary referral hospital. Clin. Infect. Dis. 46, 42-49 (2008).

8 Clinical and Laboratory Standards Institute (CLSI). Methods for dilution antimicrobial susceptibility tests for bacteria that grow aerobically. M7-A7; Approved standardSeventh Edition. (Clinical and Laboratory Standards Institute, Wayne, PA, 2006). 